\title{
Detection and Molecular Monitoring of Minimal Residual Diseases in Acute Myeloid Leukemias
}

\section{Cameron Yin}

Department of Hematopathology, The University of Texas M.D. Anderson Cancer Center, Houston, TX 77030, USA

Acute myeloid leukemias (AML) are a group of genetically heterogeneous diseases characterized by abnormal proliferation of hematopoietic precursors and disruption of normal hematopoiesis. The third and fourth editions of the World Health Organization classification of myeloid neoplasms incorporated genetic information into AML diagnostic algorithms. The recent rapid emergence of molecular markers that play a role in leukemogenesis has revolutionized the diagnosis, risk stratification, target therapy, and monitoring of minimal residual disease (MRD) of AML.

The term "minimal residual disease" refers to the persistence of residual neoplastic cells below the threshold of conventional morphologic detection. Monitoring of MRD allows for early detection of hematologic relapse and timely therapeutic intervention, and has significantly improved clinical outcome of many hematopoietic neoplasms. The detection of MRD in AML can be based on one of three major classes of molecular genetic abnormalities: chromosomal translocations/inversions, gene mutations and gene overexpressions.

Examples of AML with recurrent chromosomal translocations/ inversions include $\mathrm{t}(8 ; 21)(\mathrm{q} 22 ; \mathrm{q} 22) / R U N X 1-R U N X 1 T 1$, inv(16) (p13.1q22) or $\mathrm{t}(16 ; 16)(\mathrm{p} 13.1 ; \mathrm{q} 22) / C B F B-M Y H 11$ and $\mathrm{t}(15 ; 17)$ (q22;q21)/PML-RARA, each results in the formation of a fusion gene that plays a role in leukemogenesis. Although at initial diagnosis, these abnormalities can be detected by conventional cytogenetic analysis, fluorescence in situ hybridization (FISH), or quantitative reverse transcriptase-polymerase chain reaction (qRT-PCR), karyotyping (sensitivity 5\%) and FISH (sensitivity $0.5 \%$ ) lack the sensitivity and ease of automation of PCR for MRD analysis. qRT-PCR offers a much higher sensitivity $\left(1 \times 10^{-5}\right)$, and has become the most commonly used method for MRD detection. Association of molecular remission and clinical remission has been well documented in AML with inv $(16) / t(16 ; 16)$ and AML with $\mathrm{t}(15 ; 17)$, but the correlation is controversial for AML with $\mathrm{t}(8 ; 21)$. Some reported that the RUNX1-RUNX1T1 fusion transcript persisted in bone marrow samples from AML patients with $t(8 ; 21)$ who were in long-term clinical remission, whereas others showed that complete molecular remission could be achieved with intensive therapy and was associated with long-term clinical remission. Nevertheless, when caution in results interpretation is practiced, especially if a "cut-off" value that can predict clinical outcome is set, quantitative molecular monitoring of RUNX1-RUNX1T1 fusion transcript may be useful in MRD testing in AML patients with $t(8 ; 21)$.

Molecular screening identifies genetic aberrations in over $85 \%$ of AML patients with normal cytogenetics (CN-AML), the best studied are mutations of NPM1 (nucleophosmin1) and FLT3 (fms-related tyrosine kinase 3). Sanger sequencing has limited value in MRD analysis due to its low sensitivity (20\%). Pyrosequencing has a sensitivity of $1-5 \%$ and also allows for assessment of the ratio of mutated to unmutated clones. It works best for mutation analysis of genes with known "hot-spot" point mutations. Some gene mutations, such as the $4 \mathrm{bp}$ insertion of NPM1 and internal tandem duplication (ITD) of FLT3, can be detected by PCR followed by capillary electrophoresis (PCR-CE, sensitivity 1-5\%). For genes with known single base pair mutations, quantitative allele-specific PCR can detect the presence of a mutation as low as $0.01 \%$ of the template DNA, but two pairs of primers and/or probes need to be designed for each individual point mutation.

NPM1 mutations occur in 25-35\% of adult AML overall and 45$65 \%$ of CN-AML. In the absence of FLT3-ITD, NPM1 mutations have been associated with a favorable clinical outcome. More than 50 NPM1 mutant variants have been described, most of which consist of 4 bp insertions in exon 12. NPM1 mutations appear to be very stable at relapse, making it one of the best markers for MRD monitoring in CN-AML. FLT3-ITD occur in 20-25\% of AML overall and 35-40\% of $\mathrm{CN}-\mathrm{AML}$, and has been associated with inferior clinical outcome. The role of FLT3-ITD in MRD monitoring, on the other hand, has been debated. It has been regarded as a relatively unstable marker that can be lost at relapse. However, a subsequent study using a large cohort of patients showed that FLT3-ITD was lost in only $<5 \%$ of the patients at relapse. Therefore, when used with caution, FLT3-ITD may serve as a useful marker in AML patients with no other specific MRD marker and in patients receiving FLT3 inhibitors. Both NPM1 mutations and FLT3-ITD can be detected with PCR-CE. This approach yields semi quantitative results and can detect most of the known mutations, but has a limited sensitivity of 1-5\%. Quantitative real-time (RQ)-PCR provides quantitative results and offers a much higher sensitivity $(0.01 \%)$, and is regarded as the best method for MRD monitoring in NPM1-mutated cases, but it may miss some rare NPM1 mutant variants. It is labor intensive to design primers/probes for FLT3-ITD as the FLT3-ITD is highly variable in location and size.

In addition to structural genetic abnormalities, changes in the level of expression of certain genes have also been shown to have prognostic impact on AML. WT1 (Wilms tumor 1) mutations are found in approximately $10 \%$ of $\mathrm{CN}$-AML with conflict prognostic significance. In addition, WT1 has been shown to be overexpressed in over $90 \%$ of AML cases, and its overexpression has been associated with poor clinical outcome. It was reported that high level of WT1 expression in both bone marrow and peripheral blood as assessed by qRT-PCR correlated with disease burden in AML patients, and a rise in WT1 expression preceded hematological relapse, suggesting a role of WT1 in MRD monitoring.

*Corresponding author: C. Cameron Yin, Department of Hematopathology, The University of Texas M.D. Anderson Cancer Center, Houston, TX 77030, USA E-mail: cyin@mdanderson.org

Received February 25, 2012; Accepted March 02, 2012; Published March 04, 2012

Citation: Yin CC (2012) Detection and Molecular Monitoring of Minimal Residual Diseases in Acute Myeloid Leukemias. J Mol Biomark Diagn 2:e106. doi:10.4172/2155-9929.1000e106

Copyright: (c) 2012 Yin CC. This is an open-access article distributed under the terms of the Creative Commons Attribution License, which permits unrestricted use, distribution, and reproduction in any medium, provided the original author and source are credited 
Citation: Yin CC (2012) Detection and Molecular Monitoring of Minimal Residual Diseases in Acute Myeloid Leukemias. J Mol Biomark Diagn 2:e106. doi:10.4172/2155-9929.1000e106

Page 2 of 2

In summary, disease-specific fusion genes are the most useful markers for MRD monitoring. In CN-AML, gene mutations (e.g. NPM1, FLT3) or overexpressions (e.g. WT1) can be used for MRD assessment.

However, suitable molecular genetic markers are not yet available in approximately $20 \%$ of AML patients. This mandates the necessities to discover new biomarkers that have a role in leukemogenesis, risk stratification, disease monitoring and target therapy so that the panel of MRD markers could be expanded. 